\title{
Gain scheduling using the Youla parameterization
}

\author{
Niemann, Hans Henrik; Stoustrup, Jakob
}

Published in:

Proceedings of The 38th IEEE Conference on Decision and Control

Link to article, DOI:

10.1109/CDC.1999.831265

Publication date:

1999

Document Version

Publisher's PDF, also known as Version of record

Link back to DTU Orbit

Citation (APA):

Niemann, H. H., \& Stoustrup, J. (1999). Gain scheduling using the Youla parameterization. In Proceedings of The 38th IEEE Conference on Decision and Control (Vol. 3, pp. 2306-2311). IEEE.

https://doi.org/10.1109/CDC.1999.831265

\section{General rights}

Copyright and moral rights for the publications made accessible in the public portal are retained by the authors and/or other copyright owners and it is a condition of accessing publications that users recognise and abide by the legal requirements associated with these rights.

- Users may download and print one copy of any publication from the public portal for the purpose of private study or research.

- You may not further distribute the material or use it for any profit-making activity or commercial gain

- You may freely distribute the URL identifying the publication in the public portal

If you believe that this document breaches copyright please contact us providing details, and we will remove access to the work immediately and investigate your claim. 


\section{Gain Scheduling using the Youla Parameterization}

\author{
Henrik Niemann \\ Department of Automation \\ Technical University of Denmark \\ Building 326 \\ DK-2800 Lyngby, Denmark. \\ E-mail: hhn@iau.dtu.dk
}

\author{
Jakob Stoustrup \\ Dept. of Control Engineering \\ Aalborg University \\ Fr. Bajersvej 7C \\ DK-9220 Aalborg $\varnothing$, Denmark \\ E-mail: jakob@control.auc.dk \\ URL: www.control.auc.dk/ jakob
}

\begin{abstract}
Gain scheduling controllers are considered in this paper. The gain scheduling problem where the scheduling parameter vector $\theta$ cannot be measured directly, but needs to be estimated is considered. An estimation of the scheduling vector $\theta$ has been derived by using the Youla parameterization. The use of the Youla parameterization in connection with estimation of $\theta$ directly gives a validation method for the estimate of $\theta$. The validation part is an integrated part of the estimation method. This will make it possible to estimate the parameter vector $\theta$ very precisely. This is important in connection with $\mathcal{H}_{\infty}$ gain scheduling controllers.
\end{abstract}

Keywords: Gain scheduling, Youla parameterization, parameter estimation.

\section{Introduction}

Gain scheduling control has been an attractive approach to increase performance/robustness for systems with nonlinearity, parameter variations, uncertainties etc. The gain scheduling approach is a quite good alternative to using a single robust controller designed based on the small gain theorem. In the cases where it is possible to detect a number of operating points, a reduction of the conservatism can be obtained by designing robust controllers around each operating point and then switch between the controllers according to some gain scheduling rules. This approach has been described in e.g. [2].

Gain scheduling techniques are motivated by the large number of control applications that have significant nonlinearities which can not always be handled well by linear control design techniques. Quite a number of papers dealing with gain scheduling control for non-linear systems has emerged in recent years, see e.g. $[4,9]$ to mention a few important contributions.
However, also for important classes of linear parameter varying (LPV) systems, gain-scheduling techniques offer a good approach to get a global controller structure which is the subject of this paper. This can be achieved either by discretizing the operating range or by continuously updating the controller parameters, in which case the functionality is similar to adaptive controller schemes.

\section{Gain Scheduling Controllers}

A short introduction to gain scheduling controllers is given in the following. Let us consider a linear parameter-varying (LPV) system of the form:

$$
\begin{aligned}
& \dot{x}(t)=A(\theta(t)) x(t)+B(\theta(t)) u(t) \\
& y(t)=C(\theta(t)) x(t)+D(\theta(t)) u(t)
\end{aligned}
$$

where $\theta(t)$ is a vector of time-varying system parameters with a bounded derivative and $A(\cdot), B(\cdot), C(\cdot)$ and $D(\cdot)$ are fixed continuous functions of $\theta$.

It is assumed that a control design method for linear time invariant systems is chosen such that for each fixed value of $\theta(\cdot)$, i.e. $\theta\left(t_{i}\right)$, a fixed controller can be computed as $K\left(\theta\left(t_{i}\right)\right)=$ $\left(A_{c}\left(\theta\left(t_{i}\right)\right), B_{c}\left(\theta\left(t_{i}\right)\right), C_{c}\left(\theta\left(t_{i}\right)\right), D_{c}\left(\theta\left(t_{i}\right)\right)\right)$

Now, assuming that $\dot{\theta}(t)$ is sufficiently small, from continuity of the matrix variables, it. follows that a performance close to that designed for the system (1) can be obtained by the controllers $K\left(\theta\left(t_{i}\right)\right)$, provided the parameters $\theta\left(t_{i}\right)$ are updated sufficiently often. In the limiting case, i.e. by continuously updating $\theta$, an adaptive control scheme is obtained which is also known to have guaranteed stability and performance properties, provided the parameter change is sufficiently slow.

One issue that is not always emphasized sufficiently in literature in view of its practical importance is concerned with the implementation of gain scheduled controllers. A popular approach to implement gain scheduled controllers is shown in Figure 1. 


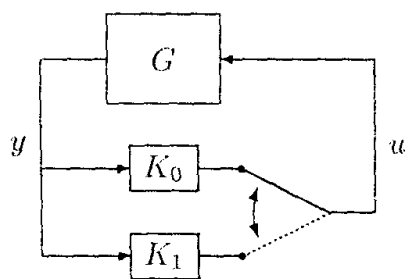

Figure 1: Gain scheduling by switching between two controllers

The basic idea in the architecture in Figure 1 is that the state of each controller is continuously updated by the measurement signal $y$ to ensure a smooth transition from controller $K_{0}$ to $K_{1}$ and back. (This approach can be extended to the socalled bumpless transfer approach.)

However, the above scheme only works in the case where each of the two controllers $K_{0}$ and $K_{1}$ are (open loop) stable which is usually the case in classical control systems. However, several modern control design techniques such as e.g. $\mathcal{H}_{\infty}$ theory, often produce unstable controllers. Such controllers can not be implemented in classical gain scheduling schemes.

This problem is one of the motivations for deriving a more general scheme than switching for gain scheduling such as the one given below.

\section{Youla Parameterization of Controllers and Systems}

The Youla parameterization of controllers and systems will shortly be considered in this section. Let us consider a system in (1) for fixed $\theta, \theta(t)=\theta_{0}$ given by the following state space representation:

$$
G_{y u}(s)=\left[\begin{array}{c|c}
A & B_{u} \\
\hline C_{y} & D_{y u}
\end{array}\right]
$$

Now, let a coprime factorization of the system $G_{y u}(s)=C_{y}(s I-A)^{-1} B_{u}+D_{y u}$ from (2) and a stabilizing controller $K(s)$ be given by:

$$
\begin{aligned}
& G_{y u}=N M^{-1}=\tilde{M}^{-1} \tilde{N} \\
& K=U V^{-1}=\tilde{V}^{-1} \tilde{U}
\end{aligned}
$$

where the eight matrices $N, M, \tilde{N}, \tilde{M}, U, V, \tilde{U}$ and $\tilde{V} \in \mathcal{R} \mathcal{H}_{\infty}$ must satisfy the double Bezout equation given by, see $[10,12]$ :

$$
\begin{gathered}
{\left[\begin{array}{cc}
\tilde{V} & -\tilde{U} \\
-\tilde{N} & \tilde{M}
\end{array}\right]\left[\begin{array}{cc}
M & U \\
N & V
\end{array}\right]=} \\
{\left[\begin{array}{cc}
M & U \\
N & V
\end{array}\right]\left[\begin{array}{cc}
\tilde{V} & -\tilde{U} \\
-\tilde{N} & \tilde{M}
\end{array}\right]=\left[\begin{array}{ll}
I & 0 \\
0 & I
\end{array}\right]}
\end{gathered}
$$

Based on the above coprime factorization of the system $G_{y u}(s)$ and the controller $K(s)$, we can give a parameterization of all controllers that stabilize the system in terms of a stable parameter $Q(s)$, i.e. all stabilizing controllers are given by [10]:

$$
K(Q)=U(Q) V(Q)^{-1}
$$

where

$$
U(Q)=U+M Q, V(Q)=V+N Q, Q \in \mathcal{R} \mathcal{H}_{\infty}
$$

or by using a left factored form:

$$
K(Q)=\dot{V}(Q)^{-1} \hat{U}(Q)
$$

where

$$
\tilde{U}(Q)=\tilde{U}+Q \dot{M}, \tilde{V}(Q)=\tilde{V}+Q \tilde{N}, Q \in \mathcal{R} \mathcal{H}_{\infty}
$$

Using the Bezout equation, the controller given either by (5) or by (6) can be realized as an LFT (Linear Fractional Transformation) in the parameter $Q$,

$$
K(Q)=\mathcal{F}_{l}\left(J_{K}, Q\right)
$$

where $J_{K}$ is given by

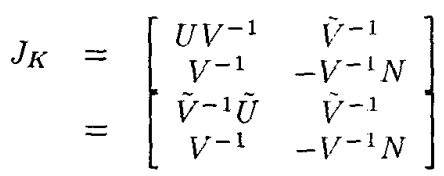

In the same way, it is possible to derive a parameterization in terms of a stable parameter $S$ of all systems that are stabilized by one controller. The parameterization is given by [10]:

$$
G(S)=N(S) M(S)^{-1}
$$

where

$$
N(S)=N+V S, M(S)=M+U S, S \in \mathcal{R} \mathcal{H}_{\infty}
$$

or by using a left factored form:

$$
G(S)=\tilde{M}(S)^{-1} \tilde{N}(S)
$$

where

$$
\tilde{N}(S)=\tilde{N}+S \tilde{V}, \tilde{M}(S)=\tilde{M}+S \tilde{U}, S \in \mathcal{R} \mathcal{H}_{\infty}
$$

An LFT representation of (9) or (10) is given by:

$$
G(S)=\mathcal{F}_{l}\left(J_{G}, S^{\prime}\right)
$$


where $J_{G}$ is given by

$$
J_{G}=\left[\begin{array}{cc}
N M^{-1} & \tilde{M}^{-1} \\
M^{-1} & -M^{-1} U
\end{array}\right]
$$

It is possible to give an interpretation of both the $Q$ parameter as well as the $S$ parameter in the above parameterization. In the following, we need the interpretation of the $S$ parameter. Let the real system from (1) be given by $G_{y u}(\theta(t))$.

(Here, and below with some abuse of notation, we shall not distinguish between transfer functions and their equivalent time-domain operators. The dependence of $\theta(t)$ is taken to be understood in the sense $\left.G_{y u}(\theta(t))=\left.\mathcal{L}^{-1}\left\{G_{y u}(\theta(\tau))\right\}\right|_{\theta(\tau)=\theta(t)}.\right\}$

The connection between the real system described by $G_{y u}(\theta(t))$ and the $S$ parameter is given by:

$$
\begin{aligned}
S= & V^{-1}\left(I-G_{y u}(\theta(t)) U V^{-1}\right)^{-1} \\
& \times\left(G_{y u}(\theta(t)) M-N\right) \\
= & \left(\bar{M} G_{y u}(\theta(t))-\tilde{N}\right) \\
& \times\left(I-\tilde{V}^{-1} \tilde{U} G_{y u}(\theta(t))\right)^{-1} \tilde{V}^{-1}
\end{aligned}
$$

It is quite clear from (13) that $S\left(\theta_{0}\right)=0$, since $G_{y u}\left(\theta_{0}\right)=N M^{-1}=\tilde{M}^{-1} \tilde{N}$. Further, if it is possible to describe $G_{y u}(\theta(t))$ as an LFT of the parameter vector $\theta(t)$, it is possible to give a more explicit connection between the parameter vector $\theta(t)$ and $S$. Let the system $G_{y u}(\theta(t))$ be described by an LFT of a nominal system and the parameter vector, $\theta(t)$, that describe the system variation,

$$
G_{y u}(\theta(t))=\mathcal{F}_{u}\left(G_{p a r}, \theta(t)\right)
$$

where

$$
G_{p a r}=\left[\begin{array}{ll}
G_{z w} & G_{z u} \\
G_{y w} & G_{y u}
\end{array}\right]
$$

Assume that the $\theta(t)$ vector is known, which makes it possible to calculate $S$ as function of $\theta(t)$. The relation between $S$ and $\theta(t)$ is then given by: [7]:

$$
S(\theta(t))=T_{3} \theta(t)\left(I-T_{1} \theta(t)\right)^{-1} T_{2}
$$

where $T_{i} \in \mathcal{R} \mathcal{H}_{\infty}$ are given by $T_{1}=G_{z w}+$ $G_{z u} U \hat{M} G_{y w}, T_{2}=G_{z u} M$ and $T_{3}=\tilde{M} G_{y w}$.

Let us close this section by considering the calculation of $S(\theta(t))$. For the calculation of $S(\theta(t))$, let us consider the closed loop system consisting of $G_{y u}(S)$ and $K(Q)$, shown in Figure 2.

The open loop transfer function $T_{o l, r s}$ from $s$ to $r$ is given by, [10]:

$$
\begin{aligned}
T_{o l, r s}= & -V^{-1} N+V^{-1} G_{y u}(S(\theta(t))) \\
& \times\left(I-\dot{V}^{-1} \tilde{U} G_{y u}(S(\theta(t)))\right)^{-1} \tilde{V}^{-1}
\end{aligned}
$$

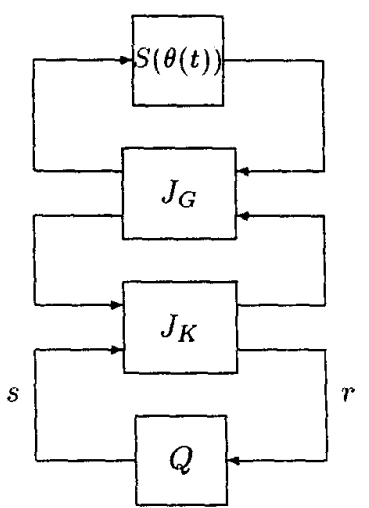

Figure 2: Closed loop transfer function of $G_{y u}(S)$ and $K(Q)$.

where $G_{y u}(S(\theta(t)))=N M^{-1}+\tilde{M}^{-1} S(\theta(t))(I+$ $\left.M^{-1} U S(\theta(t))\right)^{-1} M^{-1}=\left(N+N M^{-1} U S(\theta(t))+\right.$ $\left.\tilde{M}^{-1} S(\theta(t))\right)(M+U S(\theta(t)))^{-1}$. Hence, simply:

$$
T_{o l, r s}=S(\theta(t))
$$

The closed loop transfer function $T_{c l, r s}$ is given by:

$$
T_{c l, r s}=S(\theta(t))(I-Q S(\theta(t)))^{-1}=\tilde{S}(\theta(t))(17)
$$

From (16) it is seen that $S(\theta(t))$ is the open loop transfer function between the controller variables $s$ and $r$. It is therefore possible to calculate or estimate $S(\theta(t))$ directly from accessible variables.

Based on (16) and (17), it is now possible to estimate $S(\theta(t))$ or $\tilde{S}(\theta(t))$ by using the input/output variables $(s, r)$.

\section{Gain Scheduling with unknown $\theta$}

Using a gain scheduling controller based on a parameter vector $\theta(t)$ that cannot be measured directly, requires an estimation of the parameter vector. If the switch in the gain scheduling controller should be based on an estimated parameter vector $\theta(t)$ instead of the real parameter vector $\theta(t)$. it will require a very good estimate of $\theta(t)$ to prevent loss of performance in the closed loop system. Today, there exists a large number of methods for parameter identification in both open loop as well as in closed loop systems, see e.g. $[3,5,6,11]$ and the references herein. The identification problem in connection with gain scheduling controllers is a closed-loop identification problem. This closed-loop identification problem can be transformed into an open-loop problem by using the dual Youla parameterization of all systems stabilized by a given controller, as described in the section above. There exist a number 
of methods for estimation of the dual Youla parameter $S$, see e.g. $[10,11]$, i.e. the socalled Hansen scheme. These methods estimate the transfer function $S$. An estimate of $S$ will not in general be useful in the calculating of the equivalent parameter vector $\theta$, because the connection between $S$ and $\theta$ is not a simple connection, see Section 4 . Instead, we need to look at some other methods for the identification of the parameter vector $\theta(t)$.

First, it is assumed that it is possible to describe the system completely by the nominal system and the parameter vector $\theta(t)$. This means that the dimension of the parameter vector that is going to be identified is fixed. It is normal that the model structure/dimension of the parameter vectors are fixed by the designer. This means that it will not in general be possible to identify the systems exactly. However, in our case, an exact identification of the system will be possible in principle, because the dimension of the parameter vector is fixed and known.

In [3], a parameter identification method based on the Hansen scheme has been presented. Here, the system is parameterized in terms of a parameter vector and this parameter vector is then identified in closed-loop using a modified Hansen scheme. A complete algorithm for the identification of the parameter vector that minimize a quadratic index is given in [3].

Using the identification method given in [3], we will get an estimate $\hat{\theta}(t)$ of the parameter vector $\theta(t)$, that will minimize a quadratic index. Further, in our case, it is also possible to validate the identified parameter vector $\hat{\theta}(t)$, because the parameter vector consist of real parameters. The validation of $\hat{\theta}(t)$ can be done as follows.

Based on the identified parameter vector $\hat{\theta}(t)$, the real parameter is given by:

$$
\tilde{\theta}(t)=\theta_{0}+\hat{\theta}(t)
$$

where $\theta_{0}$ is the parameter vector for the nominal system. The model of the system can now be updated. Based on this updated model, a new dual Youla parameter $S(\tilde{\theta}(t))$ can be calculated by using (13). $S(\tilde{\theta}(t))$ is given by:

$$
\begin{aligned}
S(\tilde{\theta}(t))= & V^{-1}\left(I-G_{y u}(\theta(t)) U V^{-1}\right)^{-1} \\
& \left(G_{y u}(\theta(t)) M(\tilde{\theta}(t))-N(\tilde{\theta}(t))\right) \\
= & \left(\tilde{M}(\tilde{\theta}(t)) G_{y u}(\theta(t))-\tilde{N}(\tilde{\theta}(t))\right) \\
& \left(I-\tilde{V}^{-1} \tilde{U} G_{y u}(\theta(t))\right)^{-1} \tilde{V}^{-1}
\end{aligned}
$$

As a direct consequence of this equation, the identified parameter vector $\tilde{\theta}(t)$ is the optimal vector, i.e. $\tilde{\theta}(t)=\theta(t)$, if and only if

$$
S(\tilde{\theta}(t))=0
$$

If the identified model given by $G_{y u}(\tilde{\theta}(t))$ does not describe the real system well enough, i.e. the dual Youla parameter $S(\tilde{\theta}(t))$ is too large is some sense, a new identification of $\theta$ can be derived based on the new model of the system given by $G_{y u}(\dot{\theta}(t))$. The closed-loop system given in Figure 2 is then modified by making $J_{K}$ adaptive.

It is then quite clear that the identification of $\theta(t)$ (i.e. $S$ ) will depend on the previously identified parameter vector $\dot{\theta}(t)$.

For the identification of $\theta(t)$, let us consider the following simplified feedback system described by:

$$
\begin{aligned}
y & =v+G_{y u}(\theta(t)) u \\
e & =w+y \\
u & =K e
\end{aligned}
$$

where $w$ is a reference input signal and $v$ is disturbance signal that can be considered as filtered zero mean white noise.

Using the parameterization of $G_{y u}(\theta(t))$ in terms of the dual Youla parameter $S$, given by (9), in the feedback system described by (18), it can be rewritten into Figure 3.

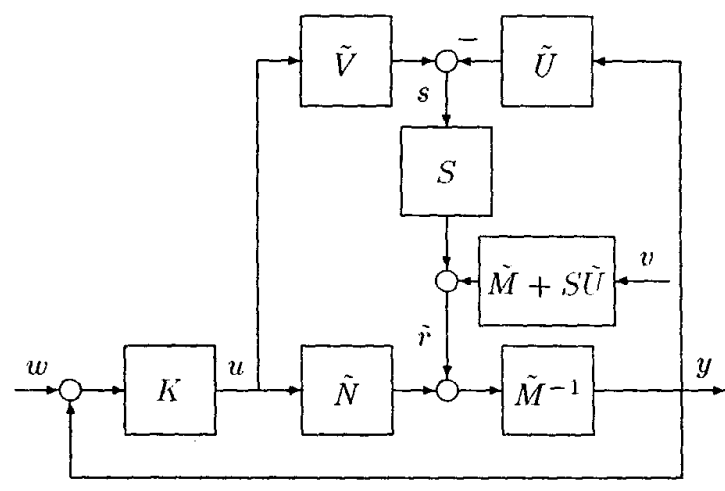

Figure 3: An alternative representation of (18).

The signals $s(t)$ and $\tilde{r}(t)$ indicated at Figure 3 are given by:

$$
\begin{aligned}
s(t) & =-\tilde{U} y+\tilde{V} u \\
& =\tilde{U} w \\
\tilde{r}(t) & =-\tilde{M} y+\tilde{N} u
\end{aligned}
$$

and further $\tilde{r}(t)$ is also given by

$$
\tilde{r}(t)=S s+(\tilde{M}+S \tilde{U}) v=r+(\tilde{M}+S \tilde{U}) v
$$

Based on (19) we get directly that the block diagram for the transfer function from $w$ and $v$ to $\tilde{r}$ is given by Figure 4.

The identification problem of $S$ is now an open-loop identification problem which can be handled using 


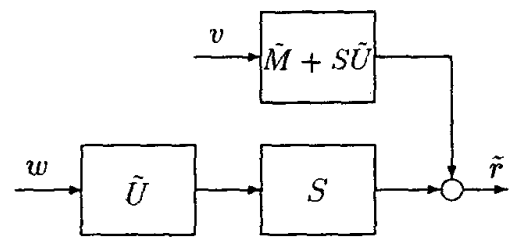

Figure 4: Open loop system for $S$.

standard methods. For doing this, let $S$ in Figure 4 be replaced by (13). The open loop system for the transfer function from $w$ to $r$ is the given by

$$
\begin{aligned}
r & =\left(V-G_{y u}(\theta(t)) U\right)^{-1}\left(G_{y u}(\theta(t)) M-N\right) \tilde{U} w \\
& =\left(V-G_{y u}(\theta(t)) U\right)^{-1}\left(G_{y u}(\theta(t)) M-N\right) s \\
& =\left(\tilde{M} G_{y u}(\theta(t))-\tilde{N}\right)\left(\tilde{V}-\tilde{U} G_{y u}(\theta(t))\right)^{-1} \tilde{U} w \\
& =\left(\tilde{M} G_{y u}(\theta(t))-\tilde{N}\right)\left(\tilde{V}-\tilde{U} G_{y u}(\theta(t))\right)^{-1} s
\end{aligned}
$$

The above equation is shown in Figure 5.

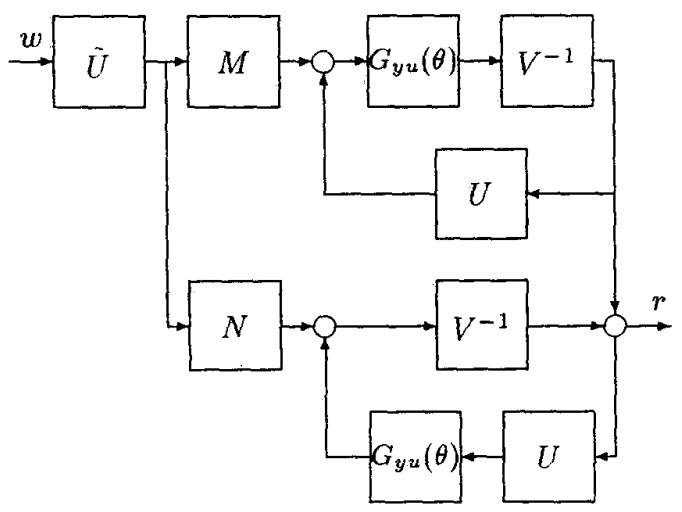

Figure 5: Open loop system for $S$.

The gradient of $r$ with respect to $\theta$ can now be found. Let the output $r(\theta)$ be given as, [3]:

$$
\begin{aligned}
r(\theta) & =\tilde{M} r_{1}(\theta)-\tilde{N} r_{2}(\theta) \\
r_{1}(\theta) & =G_{y u}(\theta) \tilde{V}^{-1}\left(s+\tilde{U} r_{1}(\theta)\right) \\
r_{2}(\theta) & =\tilde{V}^{-1}\left(s+\tilde{U} G_{y u}(\theta) r_{2}(\theta)\right)
\end{aligned}
$$

The gradient of $r(\theta)$ with respect to $\theta_{j}$ satisfies for $j=1, \cdots, n_{\theta}$

$$
r_{\theta_{j}}^{\prime}(\theta)=\tilde{M} r_{1, \theta_{j}}^{\prime}(\theta)-\tilde{N} r_{2, \theta_{j}}^{\prime}(\theta)
$$

where

$$
\begin{aligned}
r_{1, \theta_{j}}^{\prime}(\theta)= & G_{y u, \theta_{j}}^{\prime}(\theta) \tilde{V}^{-1}\left(s+\tilde{U} r_{1}(\theta)\right) \\
& +G_{y u}^{\prime}(\theta) r_{1, \theta_{j}}^{\prime}(\theta) \\
r_{2, \theta_{j}}^{\prime}(\theta)= & \tilde{V}^{-1} \tilde{U}\left(G_{y u, \theta_{j}}^{\prime}(\theta) r_{2}(\theta)\right. \\
& \left.+G_{y u}(\theta) r_{2, \theta_{j}}^{\prime}(\theta)\right)
\end{aligned}
$$

where $G_{y u, \theta_{j}}^{\prime}(\theta)$ is the derivative of $G_{y u}(\theta)$ with respect to $\theta_{j}$. $n_{\theta}$ is the number of parameters in the $\theta$ vector.

It is now possible to derive an estimate of $\theta$ based on e.g. a minimization of a quadratic index.

The derivation of the gradient of $r(\theta)$ with respect to $\theta$ has been based on the equation for $S$ given by (13). However, it is possible to rewrite the (13) which might in some cases simplifies the calculation of the gradient of $r(\theta)$. We have that $S(\theta)$ can also be described by:

$$
S(\tilde{\theta}(t))=\tilde{N}(\theta(t)) M(\tilde{\theta}(t))-\tilde{M}(\theta(t)) N(\tilde{\theta}(t))
$$

From this equation, we get directly that gradient of $r(\theta)$ with respect to $\theta_{j}$ for $j=1, \cdots, n_{\theta}$ is given by

$$
\begin{aligned}
r_{\theta_{j}}^{\prime}(\theta) & =S_{\theta_{j}}^{\prime}(\tilde{\theta}(t)) s \\
& =\left(\tilde{N}_{\theta_{j}}^{\prime}(\theta(t)) M(\tilde{\theta}(t))-\tilde{M}_{\theta_{j}}^{\prime}(\theta(t)) N(\tilde{\theta}(t))\right) s
\end{aligned}
$$

where $\tilde{N}_{\theta_{j}}^{\prime}(\theta(t))$ and $\tilde{M}_{\theta_{j}}^{\prime}(\theta(t))$ are the derivatives of $\tilde{N}(\theta(t))$ and $\tilde{M}(\theta(t))$, respectively, w.r.t. $\theta_{j}$.

The above equation can be very useful when it is possible to give explicit equations for the coprime factors of $G_{y u}$ as function of $\theta$.

Finally, let us give the gain scheduling controller based on an estimation of the parameter vector $\theta$. Let the gain scheduling controller for each fixed value of the estimated parameter vector $\tilde{\theta}(\cdot)$, i.e. $\tilde{\theta}\left(t_{i}\right)$, a fixed controller is given by $K\left(\tilde{\theta}\left(t_{i}\right)\right)=$ $\left(A_{c}\left(\tilde{\theta}\left(t_{i}\right)\right), B_{c}\left(\tilde{\theta}\left(t_{i}\right)\right), C_{c}\left(\tilde{\theta}\left(t_{i}\right)\right), D_{c}\left(\tilde{\theta}\left(t_{i}\right)\right)\right)$.

The continuous-time gain scheduling controller $K\left(\tilde{\theta}\left(t_{i}\right)\right)$ is based on an estimation of the parameter vector $\theta$. Again, if it is assumed that $\dot{\theta}(t)$ is sufficiently small, it follows that a performance close to that designed for the system (1) can be obtained by the controllers $K\left(\tilde{\theta}\left(t_{i}\right)\right)$, provided the parameters $\tilde{\theta}\left(t_{i}\right)$ are updated sufficiently often, and $\tilde{\theta}\left(t_{i}\right)$ is close to the real parameter vector $\theta(t)$. This gain scheduling controller based on an estimation of the parameter vector $\theta$ can also be considered as a controller including an adaptive tuning of the parameter vector $\tilde{\theta}$. Note that the structure of the problem makes it possible to evaluate $\tilde{\theta}$ directly by using $S$, because $\theta$ is a real parameter vector. This is in contrast to standard identification methods, where it is not known if there exists an exact parameterization of the system.

As pointed out above, it is possible directly to validate the estimate of $\theta$ by considering $S(\tilde{\theta}(t))$. If $S(\tilde{\theta}(t))$ is small in some sense, the estimate of $\theta(t)$ is close to the real parameter vector. However, it is not possible in general to use the information from $S(\tilde{\theta}(t))$ directly in connection with evaluation of the performance of the closed loop system. The reason 
is that the dual Youla parameter $S$ can only be considered from the control input/output signals and not from the external input/output signals, see [7]. Instead, we need to consider the transfer function for the closed loop directly. Let us consider an extended system description of (1) given by:

$$
\begin{aligned}
& z=G_{z d}(\theta(t)) d+G_{z u}(\theta(t)) u \\
& y=G_{y d}(\theta(t)) d+G_{y u}(\theta(t)) u
\end{aligned}
$$

where $d$ and $z$ are the external input and output, respectively, specifying the performance of the system. Closing the loop by using the feedback controller $K(\theta(t))=U(\theta(t)) V^{-1}(\theta(t))$ results in the following closed loop transfer function from $d$ to $z,[7,12]$

$$
\begin{aligned}
T_{z d}(\theta(t)) & =G_{z d}(\theta(t)) \\
& +G_{z u}(\theta(t)) U(\theta(t)) \tilde{M}(\theta(t)) G_{y d}^{\prime}(\theta(t))
\end{aligned}
$$

The closed-loop transfer function $T_{z d}(\theta(t))$ is optimal when we use the real parameter vector $\theta(t)$ in the controller. Using the gain scheduling controller $K\left(\tilde{\theta}\left(t_{i}\right)\right)$ based on the estimated parameter vector $\bar{\theta}(t))$, the closed-loop transfer function will then be given by:

$$
\begin{aligned}
T_{z d}\left(\tilde{\theta}\left(t_{i}\right)\right) & =G_{z d}(\theta(t)) \\
& +G_{z u}(\theta(t)) U\left(\tilde{\theta}\left(t_{i}\right)\right) \tilde{M}(\theta(t)) G_{y d}(\theta(t))
\end{aligned}
$$

How close the closed-loop transfer function $T_{z d}(\tilde{\theta}(t))$ is to the optimal/ideal closed loop transfer function $T_{z d}(\theta(t))$ depends on how close the estimate $\tilde{\theta}(t)$ is to the real parameter vector $\theta(t)$. The difference between the two closed-loop transfer function is then:

$$
\begin{aligned}
T_{e}\left(\tilde{\theta}\left(t_{i}\right)\right)= & T_{z d}(\theta(t))-T_{z d}\left(\tilde{\theta}\left(t_{i}\right)\right) \\
= & G_{z u}(\theta(t))(U(\theta(t)) \\
& \left.-U\left(\tilde{\theta}\left(t_{i}\right)\right)\right) \tilde{M}(\theta(t)) G_{y d}(\theta(t))
\end{aligned}
$$

\section{Conclusion}

A scheme for gain scheduling control based on an unknown parameter vector has been derived in this paper. It is shown how it is possible to estimate a real parameter vector by using a dual Youla parameterization of all systems stabilized by a given controller. The important issue in connection with this concept of parameter estimation is that it is possible to validate the estimate directly by the calculation of the dual Youla parameter

The derived scheme for gain scheduling controllers can also be considered as a step towards including adaption in robust controllers. If we use a continuous updating of the parameter vector $\theta$, we will have a control scheme including an adaptive part. This controller scheme can therefore be a useful concept for introducing adaption in robust controllers and in that way increase the working range for the controller compared with standard robust controllers. For doing this, we need to have some bounds on the estimation error of the estimate for $\theta$, such that it is possible to guarantee robust stability/performance of the closed-loop system. In the papers by Apkarian and Gahinet [1] and by Packard [8], an $\mathcal{H}_{\infty}$ gain scheduling method is given based on an exact knowledge of the parameter vector $\theta$. If it is not possible to get a direct measure of $\theta$, the parameter vector needs to be estimated and the presented controller scheme in this paper can be applied. However, to guarantee that the performance for the closed-loop is always satisfied, we need to have an upper bound on the estimation error for the parameter vector.

\section{References}

[1] P. Apkarian and P. Gahinet. A convex characterization of gain scheduled $\mathcal{H}_{\infty}$ controllers. IEEE Transactions on Automatic Control, 40:853-863, 1995.

[2] K. J. Aström and B. Wittenmark. Adaptive Control. Addison-Wesley, 1989.

[3] F. De Bruyne, B.D.O. Anderson, and N. Linard. The Hansen scheme revisited. In Proceedings of the 37th Conference on Decision and Control, pages 706-711, Tampa, FL, USA, 1998.

[4] D.A. Lawrence and W.J. Rugh. Gain scheduling dynamic linear controllers for a nonlinear plant. Automatica, 31:381-390, 1995.

[5] W.S. Lee, B.D.O. Anderson, and R.L. Kosut. A new approach to adaptive robust control. International Journal of Adaptive Control and Signal Processing, 7(3):183-211, 1993.

[6] L. Ljung. System identification: Theory for the user. Prentice Hall, 1987.

[7] H.H. Niemann. Application of the dual Youla parameterization. In European Control Conference, ECC'99, Karlsruhe, Germany, 1999.

[8] A. Packard. Gain scheduling via linear fractional transformation. Systems \& Control Letters, 22:79-92, 1994.

[9] J.S. Shamma and M. Athans. Analysis of gain scheduled control for nonlinear plants. IEEE Transactions on Automatic Control, 35:898-907, 1990.

[10] T.T. Tay, I.M.Y. Mareels, and J.B. Moore. High performance control. Birkhäuser, 1997.

[11] P. Van den Hof. Closed-loop issues in system identification. In 11th IFAC Symposium on System. Identification, SYSID'g7, pages 1651-1664, Kitakyushu, Japan, 1997.

[12] K. Zhou, J.C. Doyle, and K. Glover. Robust and optimal control. Prentice Hall, 1996. 